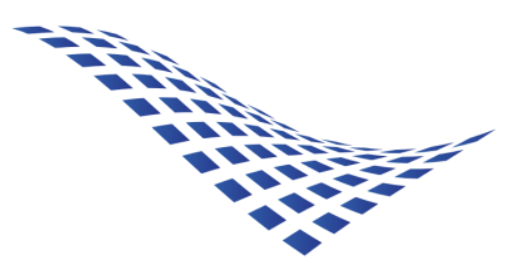

MOHAMMED VI

$P$ O L L Y T E C

U $N|V E R S| T Y$ SCIENCES ECONOMIQUES

Human Capital Index $(\mathrm{HCl})$ From Uncertainty to

Robustness of Comparisons

CAHIER

DE RECHERCHE

21-01

T. Abdelkhalek

D. Boccanfuso 


\title{
Human Capital Index (HCI) - From Uncertainty to Robustness of Comparisons
}

\author{
T. ABDELKHALEK ${ }^{1}$ and D. BOCCANFUSO*2 \\ ${ }^{1}$ Institut National de Statistique et d'Economie Appliquée - Morocco \\ ${ }^{2}$ Université Mohammed VI Polytechnique - FGSES - Morocco
}

December 22, 2020

\begin{abstract}
In 2019, the World Bank adopted an index developed by Kraay (2018), the Human Capital Index $(H C I)(\mathrm{WB}, 2019)$. It combines demographic, education, and health dimensions on a complementary statistical and econometric basis. It is used by the World Bank in the area of human development for monitoring and comparison purposes, in time and space. By construction, the $H C I$ is subject to statistical and econometric uncertainties that are not adequately captured by comparisons and are therefore, not robust.

In this paper, we propose a systematic approach taking into account these simultaneous uncertainties using a projection method. We present its practical implementation to construct confidence intervals to the $H C I$ that reflect these uncertainties. It appears that if confidence intervals overlap for two countries or for the same country over time, then comparisons would be inconclusive regardless of the ponctual estimates.
\end{abstract}

Key words : Human Capital Index (HCI); Uncertainty; Robustness; Comparisons; Projection method.

JEL Codes: C18 ; I15 ; I25

*Author for correspondence: Dorothee.BOCCANFUSO@um6p.ma 


\section{Introduction}

Within the framework of the Sustainable Development Goals $(S D G)$, it is now accepted that good health conditions and access to quality education are essential for development, especially for the new generations to ensure their optimal future contribution to society. In order to monitor progress in human development within and between countries and over time, several simple and composite indices have been developed and are regularly used, particularly in public policy decision-making. The best known is that of the United Nations Development Programme (UNDP), the Human Development Index (HDI).

More recently, the World Bank adopted a composite index proposed by Kraay (2018); the Human Capital Index (HCI). This index was constructed to approximate the expected accumulation in terms of human capital of a child born today, to be attained by age 18. Three dimensions are considered: survival, expected learning-adjusted years of school and health (WB, 2019). Its calculation is then based on demographic, health and education metrics and econometric approaches.

Since its implementation, the World Bank has used this index in two reports and calculated it for several countries. It presents implicit or explicit comparisons between countries based on the values of scores or ranks relative to the HCI. However, given the method of calculating the index, it is obviously subject to statistical and econometric uncertainty. When uncertainty is not taken into account, comparisons in time and space based on this index become therefore fragile and above all not robust. Even if Kraay (2018) underlines this problem, the solutions he proposes are not based on a systematic method. In this article, we fill this gap without questioning the relevance of the index or its method of calculation. We propose a confirmed econometric method to construct confidence intervals for the $H C I$. This is the projection method developed by Rao (1973) and used in another context by Abdelkhalek and Dufour (1998). We introduce it theoretically and then implement it numerically, using the World Bank database to compute the $H C I$.

In section 2, we conduct a brief review of the literature around the HCI. We present formally the index (subsection 2.1) followed by a critical analysis of the uncertainty to which it is subject (subsection 2.2. The methodology we propose to construct confidence intervals for the $H C I$ is presented in section 3 as well as the data that allowed its numerical implementation. We present the system of equations relating to the proposed method as well as its numerical resolution. In the last section (section 4), we report the obtained results and discuss them before concluding. 


\section{Literature review}

In 2018, the World Bank launched its first edition of the Human Capital Index. The design of this index is in line with the The Human Capital Project, which has also two other dimensions: Strengthening Measurement and Research and the Country Collaboration. In our opinion, this demonstrates the orientations and expectations of the World Bank regarding the future of this index, which has already aroused the interest of several researchers. In fact, several researchers have already taken an interest in this index.

In this context, two reports on the $H C I$ have been published using the theoretical basis developed by Kraay (2018). In 2020, the World Bank updated and improved the HCI (WB, 2020). In this last edition, it provides a profile of human capital just prior to the COVID-19 pandemic that can be used as a reference for impact analyses related to the pandemic and the mitigation policies put in place.

By 2016, to understand the gap in income per worker between Latin American countries and the United States, Caselli (2016b) already uses a proxies-based approach for three components related to health (1 dimension) and education (2 dimensions) on crosssectional data for 122 countries in 2000. These dimensions are the same as those used by Kraay (2018) in its conception of the HCI. Caselli (2016b) then uses the relationship between these three dimensions and the level of GDP per capita to identify the role of each of the gaps between these measures on differences in GDP per capita. Geographical comparisons are also conducted by distinguishing five sub-regions at the global level.

Campbell and Üngör (2020) propose a measure of human capital still based on the formulation proposed by Caselli (2016b) and Caselli (2016a) using the same three components. These authors find that it is highly correlated with the HCI (Pearson's coefficient equal to 0.945$)$ which is becoming a reference.

An alternative measure of human capital, with a philosophy close to that of the $H C I$, has been proposed by Lim et al. (2018). It is based on four dimensions, namely level of education, learning, health and survival for 195 countries. This measure was calculated for these countries between 1990 and 2016 and is updated annually.

In 2019, Angrist et al. (2019) have built another database focusing specifically on the education dimension and covering 164 countries and territories representing $98 \%$ of the world population between 2000 and 2017. This standardized database is updated annually, is openly accessible and allows for comparisons to be made. The data from this database is widely used to measure the education dimension of the $H C I$. It also makes it possible to present stylized facts and build models to analyze relationships between human capital, particularly in the quality of education dimension, and other variables of interest in terms of economic development. It is also possible to make comparisons between several groups of countries both in terms of the $H C I$ and its education-related 
dimensions. Using data from this database, Angrist et al. (2019) also develops a measure of human capital based on the quality of education and compares it to alternative measures from the Penn World Tables data.

In addition to researchers working on the design and measurement of the $H C I$, some of them build the databases needed for the calculations, while others use the $H C I$ for analysis. Indeed, in their article, Collin and Weil (2020) are not interested in the design of the $H C I$ itself but use it in a dynamic and simulation framework to analyze the effects of changes in the index on growth rates and poverty measures. They consider several scenarios for the evolution of the HCI for the 20-24 age cohort in the population.

Pasquini and Rosati (2020) estimate the HCI at the provincial level in Italy. Their objective is to capture and confirm the heterogeneity in human capital among 110 Italian provinces in 2016. The authors also propose a provincial ranking in three main groups based on the level of HCI and on the income per capita. They deduced the socioeconomic characteristics to explain these differences between groups of provinces in terms of human development.

It is reasonable to believe that at each of the stages of construction of the $H C I$ i.e. design, formulation, basic indicators, measurement of variables, parameter estimation and analysis, several sources of uncertainty can be identified. For example, in the article by Collin and Weil (2020), it is shown that any change in the level of the HCI implies a change in the level of GDP per capita and in the poverty rate. Thus, any uncertainty in the measurement of the $H C I$ will be translated de facto into uncertainty in these other indicators (GDP per capita and poverty). In general, uncertainty about the index developed by Kraay (2018) is present in all analyses and rankings based on it and can therefore be questioned. Our approach makes a significant contribution in this sense by proposing the construction of confidence intervals (measure of uncertainty) of the HCI using confirmed statistical methods. With a marginal extension, our method can be used to take into account this uncertainty on the other variables related to the $H C I$ deduced in other analyses (GDP per capita, growth rate, poverty rate, ...).

\section{$2.1 \quad H C I$ : Presentation}

The $H C I$ is a measure that is intended to be transparent and easy to understand in order to assist in the implementation of public policies related to education and/or health. This composite index combines dimensions related to health and education. It provides an estimate of the quality of human capital that a child born today can expect to obtain at the age of 18 given the health and schooling conditions in a given geographic area. The definition given by Kraay (2018) to this Human Capital Index is as follows:

"The Human Capital Index (HCI) is an international metric that benchmarks key components of human capital across countries. Measuring the 
human capital that a child born today can expect to attain by her $18^{\text {th }}$ birthday, the HCI highlights how current health and education outcomes shape the productivity of the next generation of workers. In this way, it underscores the importance for governments and societies of investing in the human capital of their citizens."

Source : Kraay (2018)

The HCI therefore aims to characterize today's decisions about the human capital of the next generation. The three components considered in the construction of the $H C I$ are :

- Survival as measured by the under-five mortality rate ;

- Years of schooling adjusted for learning achievement (or harmonized school achievement) that take into account the quality of education;

- Health, which is based on two sub-components: the rate of stunting in children under 5 years of age and the adult survival rate, defined as the proportion of 15-year-olds who will reach the age of 60 .

These three components are combined to infer the level of productivity of an adult worker as inferred by previous econometric studies. By construction, the $H C I$ is expressed as a measure of productivity relative to an optimal benchmark that results from a complete education and perfect health. This means that in a geographical area where a child born today can expect to benefit from complete health and education, i.e. without stunting, with a probability of survival to adulthood equal to one and a high quality schooling over a period of 14 years of school attendance before the age of 18, the $H C I$ would be equal to 1 (Filmer and Rogers, 2018). On the observed data, this index varies between 0.8 and 0.9 for the countries that invest the most in health and education and between 0.3 and 0.4 for the countries that invest the least in these areas $(\mathrm{WB}, 2019)$. Formally, the $H C I$ is written as follows:

$$
H C I=\text { Survival } \times \text { School } \times \text { Health }
$$

where

$$
\text { Survival }=\frac{p}{p^{*}}
$$

and $p$, the survival rate of children under 5 years of age, i.e. :

$$
p=1-T M E_{-5}
$$


where $T M E_{-5}$ is the under-five mortality rate and $p^{*}=1$;

$$
\text { School }=e^{\phi\left(S_{N G}-s^{*}\right)}
$$

with

$$
S_{N G}=E S Y \cdot \frac{T S}{s c^{*}}
$$

and with $T S$ the harminized test score, $E S Y=\sum_{a=4}^{17} E N R_{a}$ the expected number of years of schooling and where $E N R_{a}$ is the schooling rate of children of age $a$. It is therefore assumed that an education is complete and of good quality when the number of years equals $s^{*}=14$ years and with a $s c^{*}$ score of 625 points in the harmonized tests. 11

Finally, the third component is written:

$$
\text { Health }=e^{\frac{1}{2}\left(\gamma_{A S R}\left(A S R-z^{*}\right)+\gamma_{\text {Stunting }}\left(N S R-z^{*}\right)\right)}
$$

where $A S R$ is the adult survival rate, $N S R$ is the complement to one of the stunting rate and $z^{*}=1$.

The parameters $p^{*}, s^{*}, z^{*}$ and $s c^{*}$ are therefore reference values characterizing the level of human capital corresponding to a complete education and health. Kraay (2018) considers the values reported above for survival rate, years of schooling, health and harmonized score respectively.

Furthermore, $\phi, \gamma_{A S R}$ and $\gamma_{\text {Stunting }}$ are parameters that convert the components of $H C I$ into their respective contributions to productivity. Kraay (2018) calculates the $H C I$ by giving these parameters the values $0.08,0.65$ and 0.35 respectively for all countries. Thus, based on empirical data, Kraay (2018) postulates that an additional year of education increases income by $8 \%$ in adulthood $(\phi)$. In addition, it assumes that when adult survival rates increase by 10 percentage points, adult height increases by 1.9 centimetres and adult productivity increases by $3.4 \%$ for each additional centimetre $\left(\gamma_{A S R}=0.19 \times 3.4 \%=0.65 \%\right)$. Finally, it is also generally accepted that reducing stunting by 10 percentage points increases adult height by 1.02 centimetres and thus increases productivity by $3.5 \%\left(\gamma_{\text {Stunting }}=0.102 \times 3.4 \%=0.35 \%\right)$.

\subsection{About $H C I$ robustness}

Kraay (2018) itself identifies several sources of uncertainty in the calculation of the $H C I$ depending on the country. A priori each of the three components could be estimated with a standard deviation. He recommends to use of confidence intervals reflecting this incertainty for each of the components. For survival rates calculated from the under-five

\footnotetext{
${ }^{1}$ See Kraay (2018) section A2.2 for details on the methodology to determine the adjustment of education quality through harmonization of multinational tests.
} 
mortality rate, he proposes the use of the Unicef estimate $2^{2}$ For the second component, i.e. that relating to harmonized educational achievement, Kraay $(2018)$ identifies two sources of uncertainty and uses the boostrap method proposed by Patrinos and Angrist (2018) to determine confidence intervals associated with this measure. For the third component, it jointly uses estimates from the United Nations Development Programme (UNDP) and the Institute for Health Metrics and Evaluation (IHME) to obtain lower and upper bounds for adult survival rates.

For the sub-component on stunting of children under 5 years of age, Kraay (2018) suggests the use of interval estimates given by Unicef, the World Health Organization (WHO) and the World Bank when available. Finally, in the absence of a joint distribution of uncertainties for the different components that would allow these uncertainties to be considered simultaneously ${ }^{3}$, Kraay (2018) proposes a simple method for constructing a confidence interval for the $H C I$. This approach consists in retaining the lower or upper bounds of the components of interest. By this fact, it assumes a strong correlation between the different components of the $H C I$.

It follows in any case that if the confidence intervals of the $H C I$ constructed with the method proposed by Kraay (2018) between two countries overlap, one must be careful in interpreting this ranking and prefer the use of the $H C I$ value itself. This is especially recommended when implementing policies to increase the productivity of the next generation workers.In the update of its report, the World Bank has even chosen to no longer present the ranking of countries according to the value of the HCI but rather to report it by groups of countries where the $H C I$ is within a given range (WB, 2020). There are four reasons for this choice. The first is that often the basic indicators used to construct these composite indices may not be perfectly identical across countries. This is the case, for example, with school tests that may be taken at different ages depending on the country. In the case of the HCI, to our knowledge there are not yet reliable measures of the quality of higher education that are comparable in most countries of the world. A second limitation is that for a marginal variation in the $H C I$ score for a given country, the number of places gained (or lost) in the ranking would differ from one interval to another, depending on the density in terms of the number of countries in each interval. In other words, the number of countries in a given scoring interval may influence the observed changes in the ranking. A third reason is that the ranking by definition determines a relative position with respect to others. Thus, gains or losses that might be observed for a given country between two periods may not be clearly apparent if the order does not change. Finally, the authors of the World Bank report mention that the mission of this indicator is not to compare countries, but rather to measure the loss of productivity of a child born today when he or she reaches adulthood and to put

\footnotetext{
${ }^{2}$ Cf. https://data.unicef.org/topic/child-survival/under-five-mortality/.

${ }^{3} \mathrm{Or}$ at least the non-availability of a variance-covariance matrix for the estimators of these components.
} 
in place policies that will improve the country's situation $(\mathrm{WB}, 2020)$. Comparison over time, provided that the methodology remains the same, should therefore be preferred.

This reflection on the classification of the $H C I$ and the comparison in space leads us to question the inclusion of uncertainty in the calculation of the $H C I$ itself. Several authors have already pointed out the lack of robustness of the $H C I$ from its conception. Pasquini and Rosati (2020) underline the fact that setting the values of $\phi$ and $\gamma_{A S R}$ as proposed by Kraay (2018) can be arbitrary $4^{4}$ To account for some of this uncertainty, they conduct a limited sensitivity analysis of their results. However, their approach consists of varying the two parameters in an ad hoc way, $\phi$ and $\gamma_{A S R}$ from 0.08 and 0.65 to 1 simultaneously $5^{5}$ Their results show that the value of the $H C I$ changes substantially but that the ranking of Italian provinces is not affected by this change in the parameters. Again, this finding raises the problem of the reliability of the resulting ranking. ${ }^{6}$ Another measure of limited sensitivity, proposed by the authors, is to change the manner in which certain components related to the education dimension used in the calculation of the HCI. Here again, the authors find that the values of the HCI differ but that the positions remain broadly unchanged. In doing so, Pasquini and Rosati (2020) identify another source of uncertainty in the calculation of the $H C I$, the one related to the measurement of elementary indicators.

Angrist et al. (2019) are also aware of the uncertainty in the proposed HCI measurements and rankings by questioning the values of the education sub-component. They assume that the scores obtained by students in different countries follow asymptotically a Normal distribution and construct confidence intervals using the bootstrap method. These authors explicitly identify a source of uncertainty associated with the education dimension of the $H C I$.

Thus, as we have pointed out, Kraay (2018), the designer of the HCI, is aware of the limitation of the approach he proposes to construct the confidence intervals of the $H C I$. Indeed, it does not take into account all the uncertainty related to the estimation of the parameters $\phi, \gamma_{A S R}$ and $\gamma_{S t u n t i n g}$ and their impact on the education and health returns used in the calculation of the $H C I$.

In this article, we also highlight the existence of several sources of uncertainty in the calculation of the $H C I$ and in the consequent ranking. Our proposal is precisely to improve this issue at both theoretical and empirical levels. The confidence intervals that we construct with our method will allow us to nuance the weak comparisons resulting from the rankings between countries or over time.

\footnotetext{
${ }^{4}$ These authors only consider one dimension in the health component.

${ }^{5}$ See in section 2.1 the origin of the values of $\phi$ and $\gamma_{A S R}$ used by Kraay (2018).

${ }^{6}$ See Figure 14 p. 25 of Pasquini and Rosati (2020).
} 


\section{Methodology and data}

The uncertainty associated with the $H C I$ emanates from the three parameters $\phi, \gamma_{A S R}$ and $\gamma_{\text {Stunting }}$ which can be estimated in a classical way from an econometric method, but also from the values of the variables that enter into the calculation of each component of the HCI (TME, ESY, TS, ASR and NSR). A Bayesian design i.e. with a priori on the uncertainty relative to these eight variables would also be possible with our approach.

\subsection{Presentation of the method}

The approach we propose to capture the uncertainty around the punctual estimate of the $H C I$ through the construction of confidence intervals is inspired by Rao (1973) revisited and detailed by Abdelkhalek and Dufour (1998). It is a method based on projections of confidence regions. Formally, we consider the two sources of uncertainty underlined above, recognized by Kraay (2018) but never treated simultaneously.

Let $\beta$ be the vector of the eight sources of uncertainty identified in our case and let $C$ be the rectangular simultaneous confidence region of level $1-\alpha$ and associated with $\beta .7$ In other words, as in Abdelkhalek and Dufour (1998), we consider:

$$
P[\beta \in C] \geq 1-\alpha
$$

where $C$ is a random subset of $\mathbb{R}^{8}$ and with $0 \leqslant \alpha<1$. To any value of $\beta \in C$ corresponds a value possibly different of the $H C I$ and conversely, to any value of $H C I$ would correspond at least one value of $\beta \in C$. Note by $g(C)$ the image set of $C$ in $\mathbb{R}$ through the function $g($.$) such that g: \mathbb{R}^{8} \rightarrow \mathbb{R}$ which corresponds to the function that calculate HCI. As Rao (1973) shows, $g(C)$ is a confidence interval of level $1-\alpha$ for $g(\beta)$ and thus for the $H C I$. This is precisely the major theoretical contribution of this paper. Indeed, since $\beta \in C \Rightarrow g(\beta) \in g(C)$ then

$$
P[g(\beta) \in g(C)] \geq P[\beta \in C] \geq 1-\alpha .
$$

On the other hand for the implementation issues, let $g^{L}(C)$ and $g^{U}(C)$ be respectively the lower and upper bounds of the confidence interval of $g(\beta)$ i.e those of the $H C I$ defined as:

$$
\begin{array}{ll}
g^{L}(C)=\inf \{g(\beta): & \beta \in C\} \\
g^{U}(C)=\sup \{g(\beta): & \beta \in C\} .
\end{array}
$$

\footnotetext{
${ }^{7}$ The confidence region is rectangular since it is impossible to have an estimate of the variancecovariance matrix relative to the estimators of the eight parameters/variables considered. If such a matrix is available, ellipsoidal confidence regions can be constructed and retained at this level. For more details see the comment of Kraay (2018) (P. 51) and the solution proposed in Abdelkhalek and Dufour (1998).
} 
So, for every $\beta \in C$, we deduce from it

$$
g(\beta) \in g(C) \Rightarrow g^{L}(C) \leq g(\beta) \leq g^{U}(C)
$$

and therefore by combining (8) and (10) :

$$
P\left[g^{L}(C) \leq g(\beta) \leq g^{U}(C)\right] \geq P[g(\beta) \in g(C)] \geq P[\beta \in C] \geq 1-\alpha .
$$

Thus, given the available information, the a priori on uncertainty of the eight parameters/variables contained in $\beta$ and translated into the $C$ region, we can numerically construct a confidence interval for $g(\beta)$ i.e. for the $H C I$.

As proposed by Abdelkhalek and Dufour (1998), the numerical procedure consists in minimizing then maximizing $H C I=g(\beta)$ under the five constraints which allow to calculate the $H C I$ (equations 2 to 6 and the definition of the simultaneous confidence region $C$ of $\beta$ which is based on inequalities (two per source of uncertainty, i.e. 16 in total) 8 . This is the fundamental step characterizing the core of the implementation of the method.

To solve this highly non-linear mathematical program, we use the GAMS software. Let us specify that in this approach, the three parameters that appear in the calculation of the $H C I$ (that is $\phi, \gamma_{A S R}$ and $\gamma_{\text {Stunting }}$ ) become variables in the same way as the five other sources of uncertainty ( $T M E, E S Y, T S, A S R$ and $N S R$ ). During the resolution, a complete scan is performed on all these sources, simultaneously in the $C$ region considered, contrary to a limited sensitivity analysis on discrete values of these parameters as proposed by Kraay (2018) and Pasquini and Rosati (2020).

The following system must therefore be solved twice (minimization and maximization)

\footnotetext{
${ }^{8}$ The mathematical formulation of this system is detailed below.
} 
for each selected country for comparison.

$$
\left\{\begin{array}{l}
\operatorname{Min} / \operatorname{Max}_{\beta}[H C I(\beta)=g(\beta)] \\
\text { Under constraints: } \\
H C I(\beta)=\operatorname{Survival}(\beta) \times \operatorname{School}(\beta) \times \operatorname{Health}(\beta) \\
\text { Survival }=\frac{p}{p^{*}} \\
p=1-T M E_{-5} \\
\operatorname{School}=e^{\phi\left(S_{N G}-s^{*}\right)} \\
S_{N G}=E S Y^{\frac{T S}{s c^{*}}} \\
\text { Health }=e^{\frac{1}{2}\left(\gamma_{A S R}\left(A S R-z^{*}\right)+\gamma_{S t u n t i n g}\left(N S R-z^{*}\right)\right)} \\
p^{*}=1 \quad ; \quad s^{*}=14 \quad ; \quad z^{*}=1 \quad ; \quad s c^{*}=625 \\
\phi^{L} \leqslant \phi \leqslant \phi^{U} ; \gamma_{A S R}^{L} \leqslant \gamma_{A S R} \leqslant \gamma_{A S R}^{U} ; \gamma_{S t u n t i n g}^{L} \leqslant \gamma_{S t u n t i n g} \leqslant \gamma_{S t u n t i n g}^{U} \\
T M E^{L} \leqslant T M E \leqslant T M E^{U} ; E S Y^{L} \leqslant E S Y \leqslant E S Y^{U} ; T S^{L} \leqslant T S \leqslant T S^{U} ; \\
A S R^{L} \leqslant A S R \leqslant A S R^{U} ; N S R^{L} \leqslant N S R \leqslant N S R^{U}
\end{array}\right.
$$

with $\beta$ a vector of eight components which are $\phi, \gamma_{A S R}$ and $\gamma_{\text {Stunting }}, T M E, E S Y, T S$, $A S R$ and NSR. The 16 bounds of these parameters/variables (with the exponents $L$ and $U$ ) are known at this level from a classical or a Bayesian statistical approaches. The last three lines of the system 12 explicitly define the rectangular simultaneous confidence region relating to these eight sources of uncertainty, noted $C$ previously ${ }^{9}$

\subsection{Data}

To demonstrate that the approach proposed in this paper can be implemented and systematized, we use the database constructed by the World Bank to calculate the HCI used in the 2020 report (WB, 2020).$^{10}$ Kraay (2018) presents in detail the construction of each of the indicators and their sources.

In 2020 report, this database covers 217 jurisdictions (countries and territories) and contains a priori all the indicators needed to calculate the $H C I$. To these indicators, we have added a variable designating the region in which each country is located, based on the World Bank classification in which six regions are identified 11 This variable region allowed us to construct plausible bounds for each of the variables in our system 12

It should be noted that several values of certain indicators contained in the database were

\footnotetext{
${ }^{9}$ The system we propose here is general and remains unchanged even in the case where one or more uncertainties disappear (the lower and upper bounds would then be equal for the uncertainty concerned).

${ }^{10}$ For a detailed definition of the indicators used in the calculation of the $H C I$ and to access the raw database see https://databank .banquemondiale.org/source/human-capital-index

${ }^{11}$ See https://donnees . banquemondiale.org/

${ }^{12}$ This variable could also be used for intra-regional comparisons.
} 
found to be missing for some countries. However, given the demonstrative nature of this article, we have only retained those jurisdictions for which we had all the information for the intermediate variables in the calculation of the $H C I$. Then, the final database on which our exercise is based contains 91 countries 13

The first treatment we have done is to construct the rectangular simultaneous confidence region $(C)$ for the eight sources of uncertainty as it appears in the system 12 . To do this, we first deduced, at the level of each of the six regions, the minimum and maximum values of each of the five variables observed for each country ${ }^{14}$ These values are then considered lower and upper bounds respectively for each country in its region in our optimization program. Normally these five variables are calculated from country-specific statistical surveys and would be accompanied by appropriate standard deviations that would be used to construct confidence intervals for these same parameters. In this more methodological article, we have not gone as far as this level, especially since it is impossible to us to have these standard deviations for all countries and for all variables. ${ }^{15}$.

For the other three sources of uncertainty $\left(\phi, \gamma_{A S R}\right.$ and $\left.\gamma_{\text {Stunting }}\right)$ which are real parameters in the econometric sense, it would have been ideal, here again, to have punctual estimates and standard deviations (or ideally a variance-covariance matrix) associated with the estimation of these parameters for each country. These date would then have made it possible to construct the confidence region, $C$, more rigorously. However, as Kraay (2018) points out, this information is not available even for the punctual estimate. As noted in the section 11, for these three parameters the World Bank uses the same values for all countries (cited in WB (2019) and WB (2020)). In our case, we have considered that the value of $\phi$ is between 0.05 and 0.09 for all countries, knowing that the punctual estimate usually used is equal to 0.08 . For the other two parameters, the limits retained are 0.45 and 0.8 for $\gamma_{A S R}$ (for a punctual estimate of 0.65 ) and 0.25 and 0.45 for $\gamma_{\text {Stunting }}$ (for a punctual estimate of 0.35) ${ }^{16}$ Following this second step, the $C$ confidence region under consideration is fully delimited.

\subsection{Implementation and resolution}

The implementation consists in solving the System 12 using specialized software ${ }^{17}$ When writing the program, the first step is to import the database introduced in the section 3.2 and assigning initial values to the eight variables of the system as well as the bounds that define the $C$ region. $\sqrt{18}$ For verification, we first calculate the value of the $H C I$ for

\footnotetext{
${ }^{13}$ We focused our work on the year 2020. However, it can be replicated for any year and for any number of countries or groups of countries.

${ }^{14}$ The five variables that have been treated with this approach are: NSR, ASR, ESY, TS and $p$.

${ }^{15}$ This data processing was carried out with STATA software, version 16 .

${ }^{16}$ In the annexed Tables 2 to 5 , we reproduce the punctual estimates and bounds for the five sources of uncertainty varying according to the region of the 91 countries under consideration.

${ }^{17}$ In our case, we used the GAMS software.

${ }^{18}$ At this step, $\phi, \gamma_{A S R}$ and $\gamma_{\text {Stunting }}$ are considered as variables and not as parameters.
} 
2020 for each country based on the punctual estimates that were imported. The results obtained are exactly the same as those presented in the report WB (2020).

In the second step, all the equations of the System 12 are introduced as well as the 16 inequalities defining the confidence region of the eight variables under uncertainty as explained above. The appropriate initializations for all variables are also introduced. In a loop, the program solved twice the system for each of the 91 countries in our database. The first minimizes the HCI under all constraints and the second maximizes it. We then obtain respectively the minimum and maximum value of the $H C I$ for each of the countries considered. Figure 1 schematizes the implementation and the resolution. 
Figure 1: Schematic representation of the resolution algorithm

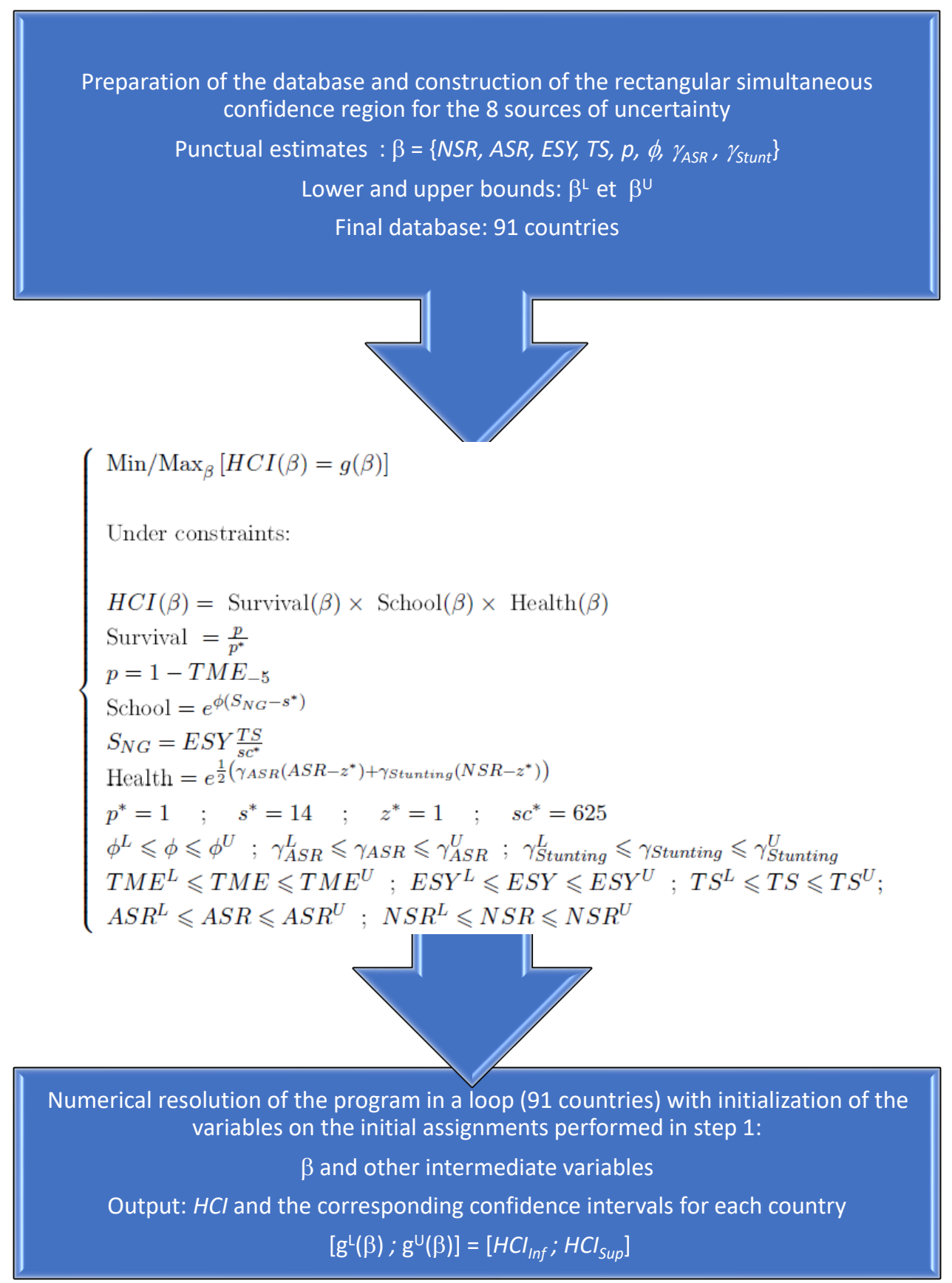

Source: Authors 


\section{Results and discussion}

Once the System 12 is solved for each country (minimization and maximization), the results for the lower and upper bounds of the $H C I$ are recovered. Table 1 presents these results in confidence intervals for the 91 countries to which we have added the calculated punctual values of the $H C I$. Countries are thus ranked in ascending order of their $H C I$ in 2020 as the World Bank continues to do.

As expected, the confidence intervals obtained are more informative for each country. They also allow comparisons to be made between countries, but especially over time 19 For example, in the case of Morocco, the punctual estimate of the HCI in 2020 is equal to 0.504. Our results show that this index would in fact lie between 0.493 and 0.514 precisely because of the various uncertainties related to its calculation, as approached and integrated into our method. A similar analysis done for a Latin American country such as Paraguay, for example, shows that this confidence interval is wider (from 0.510 to 0.542 ) for a punctual estimate equal to 0.528 . This observation can be observed for most countries of this continent. It should be noted that the intervals for Asian countries are shorter than those obtained for African or Latin American countries. This is explained by the fact that the information contained in the database for Asian countries is very close to each other (less dispersion and therefore less uncertainty) unlike African or Latin American countries where there is more dispersion in the basic data, which indicates more uncertainty.

At the level of cross-country comparisons, the results seem more reliable and robust. Indeed, on an ad hoc basis, it can be deduced that Egypt's HCI (0.494) is lower than that of Morocco (0.504) and Tunisia (0.517). However, examination of the confidence intervals for these $H C I$ for these three countries leads to nuance these rankings. It is easy to see that these confidence intervals for the $H C I$ for these three countries overlap at least two by two, which makes some comparisons ambiguous. Moreover, it is known that all the values contained in a confidence interval are statistically equivalent. Thus, the $H C I$ score of Egypt (0.494) belongs to the confidence interval constructed for the HCI of Morocco. This is the case for an infinity of other values in the confidence interval of the $H C I$ of Egypt. It also emerges that there is a very clear intersection between the confidence intervals of the $H C I$ constructed for Morocco and Tunisia. Similar results are observed by comparing the confidence intervals of the HCI constructed for Senegal and South Africa for example. For these reasons, and as noted in section 2.2, comparisons based only on punctual estimates of the $H C I$ should be conducted with caution. Sometimes comparisons based on confidence intervals lead to robust assertions. This is the case, for example, when comparing the confidence interval of Morocco's $H C I$ with that of South

\footnotetext{
${ }^{19}$ Note that our method can be used several successive years for the same country. The resulting confidence intervals can be examined to conclude about the significance of the progress made on the $H C I$ over time.
} 
Table 1: Confidence intervals of $H C I$

\begin{tabular}{|c|c|c|c|c|c|c|c|}
\hline Country & $H C I_{\text {Inf }}$ & $H C I$ & $H C I_{S u p}$ & Country & $H C I_{\text {Inf }}$ & $H C I$ & $H C I_{\text {Sup }}$ \\
\hline Chad & 0,282 & 0,300 & 0,317 & Bangladesh & 0,455 & 0,464 & 0,472 \\
\hline South Sudan & 0,267 & 0,307 & 0,334 & Zimbabwe & 0,444 & 0,467 & 0,488 \\
\hline Niger & 0,295 & 0,316 & 0,333 & Myanmar & 0,459 & 0,478 & 0,494 \\
\hline Mali & 0,307 & 0,318 & 0,329 & Honduras & 0,469 & 0,481 & 0,491 \\
\hline Liberia & 0,303 & 0,319 & 0,332 & Cambodia & 0,471 & 0,492 & 0,507 \\
\hline Nigeria & 0,335 & 0,361 & 0,381 & India & 0,489 & 0,494 & 0,498 \\
\hline Mozambique & 0,343 & 0,362 & 0,377 & Egypt & 0,480 & 0,494 & 0,508 \\
\hline Angola & 0,330 & 0,362 & 0,385 & Guyana & 0,477 & 0,495 & 0,510 \\
\hline Sierra Leone & 0,345 & 0,363 & 0,379 & $\begin{array}{l}\text { Dominican Re- } \\
\text { public }\end{array}$ & 0,489 & 0,503 & 0,515 \\
\hline $\begin{array}{l}\text { Congo, Dem. } \\
\text { Rep. }\end{array}$ & 0,344 & 0,366 & 0,385 & Morocco & 0,493 & 0,504 & 0,514 \\
\hline Guinea & 0,352 & 0,371 & 0,387 & Tajikistan & 0,481 & 0,504 & 0,525 \\
\hline Eswatini & 0,352 & 0,373 & 0,390 & Nepal & 0,490 & 0,505 & 0,519 \\
\hline Yemen, Rep. & 0,353 & 0,373 & 0,388 & Nicaragua & 0,500 & 0,508 & 0,516 \\
\hline Sudan & 0,362 & 0,377 & 0,391 & Philippines & 0,501 & 0,516 & 0,531 \\
\hline Rwanda & 0,364 & 0,380 & 0,392 & Tunisia & 0,508 & 0,517 & 0,525 \\
\hline Cote d'Ivoire & 0,362 & 0,380 & 0,398 & Paraguay & 0,510 & 0,528 & 0,542 \\
\hline Mauritania & 0,345 & 0,382 & 0,406 & Algeria & 0,527 & 0,535 & 0,542 \\
\hline Ethiopia & 0,372 & 0,383 & 0,392 & Jamaica & 0,518 & 0,535 & 0,548 \\
\hline Burkina Faso & 0,363 & 0,384 & 0,402 & Indonesia & 0,530 & 0,540 & 0,550 \\
\hline Uganda & 0,371 & 0,384 & 0,396 & El Salvador & 0,531 & 0,546 & 0,560 \\
\hline Burundi & 0,364 & 0,386 & 0,406 & Kenya & 0,527 & 0,547 & 0,565 \\
\hline Tanzania & 0,377 & 0,390 & 0,401 & $\begin{array}{l}\text { North Macedo- } \\
\text { nia }\end{array}$ & 0,551 & 0,557 & 0,563 \\
\hline Madagascar & 0,375 & 0,392 & 0,409 & Azerbaijan & 0,562 & 0,578 & 0,591 \\
\hline Zambia & 0,381 & 0,397 & 0,410 & Armenia & 0,569 & 0,579 & 0,589 \\
\hline Cameroon & 0,377 & 0,397 & 0,418 & $\begin{array}{l}\text { Bosnia and } \\
\text { Herzegovina }\end{array}$ & 0,571 & 0,580 & 0,588 \\
\hline Afghanistan & 0,387 & 0,400 & 0,413 & Moldova & 0,575 & 0,584 & 0,593 \\
\hline Lesotho & 0,375 & 0,400 & 0,423 & Ecuador & 0,585 & 0,594 & 0,603 \\
\hline Comoros & 0,360 & 0,405 & 0,434 & Kyrgyz Rep. & 0,587 & 0,597 & 0,605 \\
\hline Pakistan & 0,392 & 0,406 & 0,419 & Sri Lanka & 0,592 & 0,598 & 0,604 \\
\hline Iraq & 0,401 & 0,408 & 0,414 & Argentina & 0,592 & 0,602 & 0,612 \\
\hline Malawi & 0,395 & 0,413 & 0,429 & St. Lucia & 0,588 & 0,603 & 0,615 \\
\hline Congo, Rep. & 0,393 & 0,419 & 0,440 & Colombia & 0,592 & 0,604 & 0,616 \\
\hline Solomon Isl. & 0,409 & 0,420 & 0,430 & Peru & 0,593 & 0,605 & 0,616 \\
\hline Senegal & 0,404 & 0,420 & 0,435 & Thailand & 0,598 & 0,609 & 0,620 \\
\hline Gambia, The & 0,394 & 0,422 & 0,442 & Malaysia & 0,601 & 0,611 & 0,621 \\
\hline South Africa & 0,414 & 0,425 & 0,437 & Mexico & 0,605 & 0,613 & 0,621 \\
\hline $\begin{array}{l}\text { Papua } \quad \text { New } \\
\text { Guinea }\end{array}$ & 0,413 & 0,429 & 0,444 & Bulgaria & 0,603 & 0,614 & 0,625 \\
\hline Togo & 0,410 & 0,432 & 0,452 & Mongolia & 0,597 & 0,614 & 0,630 \\
\hline Namibia & 0,423 & 0,446 & 0,465 & Kazakhstan & 0,622 & 0,629 & 0,635 \\
\hline Haiti & 0,428 & 0,447 & 0,463 & Montenegro & 0,624 & 0,633 & 0,641 \\
\hline Ghana & 0,438 & 0,450 & 0,462 & Albania & 0,625 & 0,634 & 0,643 \\
\hline Timor-Leste & 0,432 & 0,454 & 0,472 & Turkey & 0,641 & 0,649 & 0,658 \\
\hline Vanuatu & 0,436 & 0,455 & 0,470 & China & 0,640 & 0,653 & 0,665 \\
\hline Lao PDR & 0,441 & 0,457 & 0,470 & Serbia & 0,665 & 0,677 & 0,687 \\
\hline Gabon & 0,433 & 0,458 & 0,478 & Vietnam & 0,671 & 0,690 & 0,708 \\
\hline Guatemala & 0,451 & 0,461 & 0,470 & & & & \\
\hline
\end{tabular}


Africa.

\section{Conclusion}

To monitor the progress in human development and to make comparisons over time and space, several international institutions have developed composite indices. The best known is the famous Human Development Index (HDI) of the UNDP. In 2018, in what appears to be a reaction, adopting an approach developed by Kraay (2018), the World Bank proposed its own composite index, the Human Capital Index (HCI). This last one is innovative since it focuses on the accumulation of human capital relative to future generations for children born today.

This index, like others used for international comparisons, suffers of robustness due to the lack of systematic consideration of uncertainties in its calculation. In the case of the $H C I$, the designer of the index itself recognizes this weakness. The solution proposed by Kraay (2018) is simple and ad hoc. The approach that we propose in this article largely fills this gap without questioning the relevance of the index or its calculation method.

For this purpose, we construct an econometric method based on projections of confidence regions to take into account several uncertainties related to the calculation of the $H C I$. The theoretical basis of this approach is presented in our paper as well as its numerical implementation. Using the 2020 World Bank database, we construct confidence intervals of the $H C I$ for 91 countries. These allow reliable comparisons to be made that are much more robust than those based on punctual estimates.

We believe that this approach can be systematized by the World Bank, for example, to accompany punctual estimates of the $H C I$. Confidence intervals constructed with our method allow to statistically bound the estimate of the HCI. In the case of Paraguay, for example, saying that the punctual estimate of the $H C I$ is equal to 0.528 is to be qualified since the productivity of a child born today will actually have a productivity expected today for 2038 , between $51 \%$ and $54.2 \%$. On the other hand, our method also makes it possible to nuance comparisons resulting from rankings on the base of the $H C I$ over time or between countries. On the time dimension, in the example of Paraguay, if in 2025, the calculated $H C I$ was equal to $54 \%$, with an interval similar to that of 2020, the progress made by Paraguay over this period would be considered statistically insignificant. For comparisons between countries we have shown, for example, that in 2020 between Morocco, Tunisia and Egypt, the confidence intervals overlap, which imposes a nuanced comparison. On the other hand, in other situations, our approach allows an unambiguous comparison of the situation between two countries. This is the case, for example, of Morocco in comparison with South Africa in 2020.

In a next step, the proposed method could be completed at several levels. Indeed, as 
we mentioned, in this article we have used the values proposed by Kraay (2018) for the

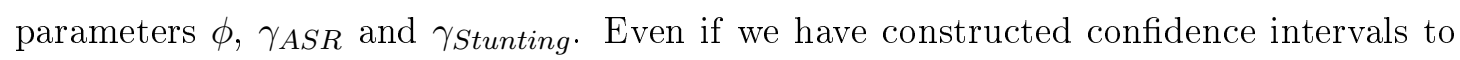
introduce uncertainty, the bounds considered remain only illustrative. It would therefore be interesting to estimate these parameters econometrically, to deduce the standard deviations for the estimated values and the corresponding confidence intervals for each country. At our level, this could only be done for a few countries for which we would have access to data. In the same perspective, the confidence region considered in this paper is a rectangular type. It would be interesting to consider ellipsoidal confidence regions at least for some parameters. For this, our method can beeasily adapted. .

The extension of our method can also be done to consider the uncertainty related to variables linked to $H C I$ deduced in other studies (GDP per capita, growth rate, poverty rate, ...). Ultimately, we plan to rapidly make systematic the use of our method to construct confidence intervals for the HCI calculated by the World Bank or other researchers, by making our GAMS and R programs freely available. 


\section{References}

Abdelkhalek, T. and Dufour, J.-M. (1998). Statistical inference for computable general equilibrium models, with application to a model of the moroccan economy. Review of Economics and Statistics, 80(4):520-534.

Angrist, N., Djankov, S., Goldberg, P. K., and Patrinos, H. A. (2019). Measuring human capital. The World Bank.

Campbell, S. G. and Üngör, M. (2020). Revisiting human capital and aggregate income differences. Economic Modelling, 91:43-64.

Caselli, F. (2016a). Accounting for Cross-Country Income Differences: Ten Years Later. World Bank.

Caselli, F. (2016b). The latin american efficiency gap. In World-Bank, editor, Understanding the income and efficiency gap in Latin America and the Caribbean, pages $33-56$.

Collin, M. and Weil, D. N. (2020). The effect of increasing human capital investment on economic growth and poverty: A simulation exercise. Journal of Human Capital, 14(1):43-83.

Filmer, D. and Rogers, H. (2018). Learning to realize educations promise. World Development Report. The World Bank.

Kraay, A. (2018). Methodology for a World Bank human capital index. The World Bank.

Lim, S. S., Updike, R. L., Kaldjian, A. S., Barber, R. M., Cowling, K., York, H., Friedman, J., Xu, R., Whisnant, J. L., Taylor, H. J., Leever, A. T., Roman, Y., Bryant, M. F., Dieleman, J., Gakidou, E., and Murray, C. J. L. (2018). Measuring human capital: a systematic analysis of 195 countries and territories, 1990-2016. The Lancet, 392(10154):1217 - 1234.

Pasquini, A. and Rosati, F. C. (2020). A human capital index for the italian provinces. IZA Discussion Paper, (13301).

Patrinos, H. A. and Angrist, N. (2018). Global Dataset on Education Quality: A Review and Update (2000-2017). The World Bank.

Rao, C. R. (1973). Linear statistical inference and its applications, volume 2. Wiley New York.

WB (2019). Le projet sur le capital humain.

WB (2020). The human capital index 2020 update - human capital in the time of COVID-19. 
Annex 


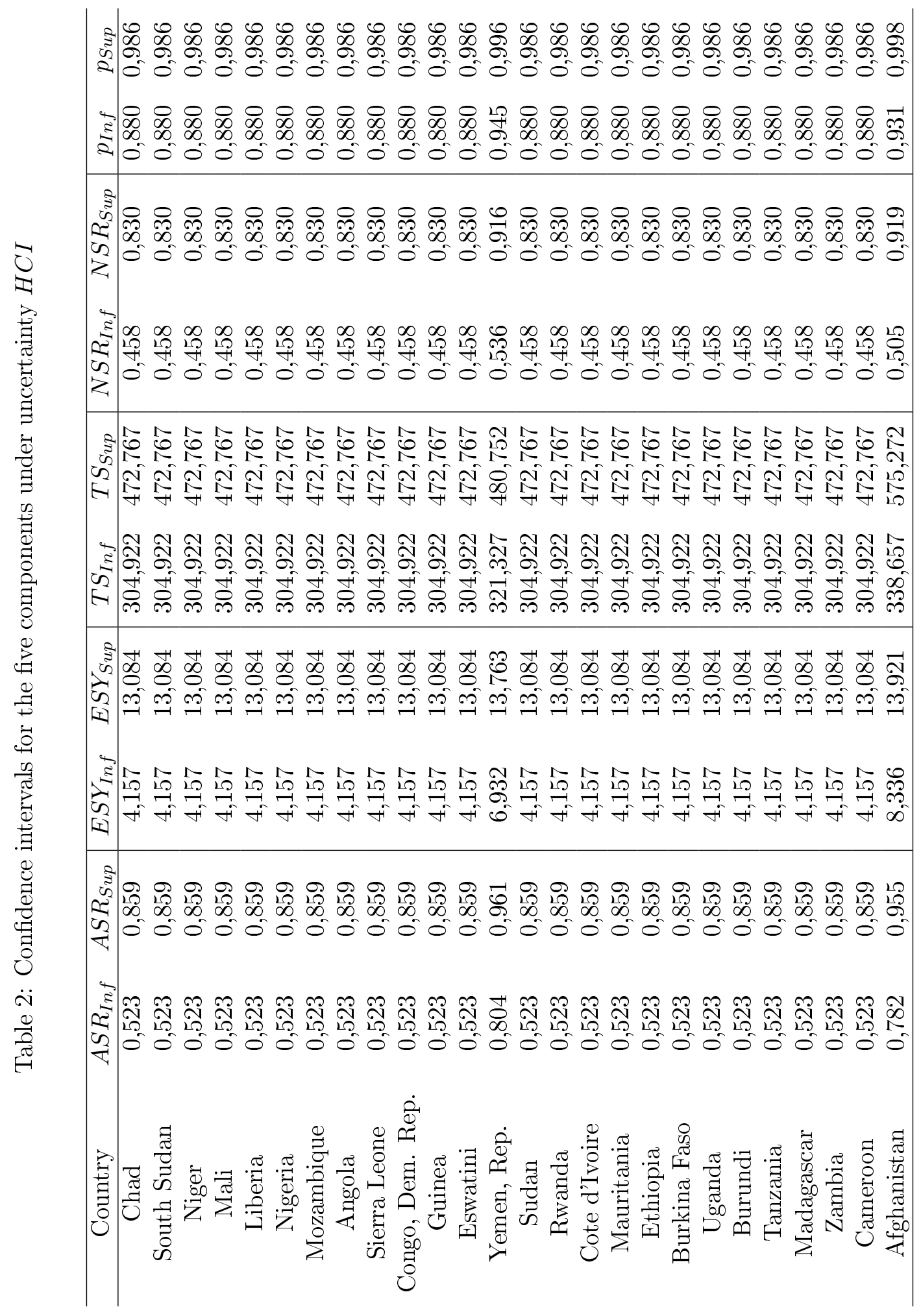




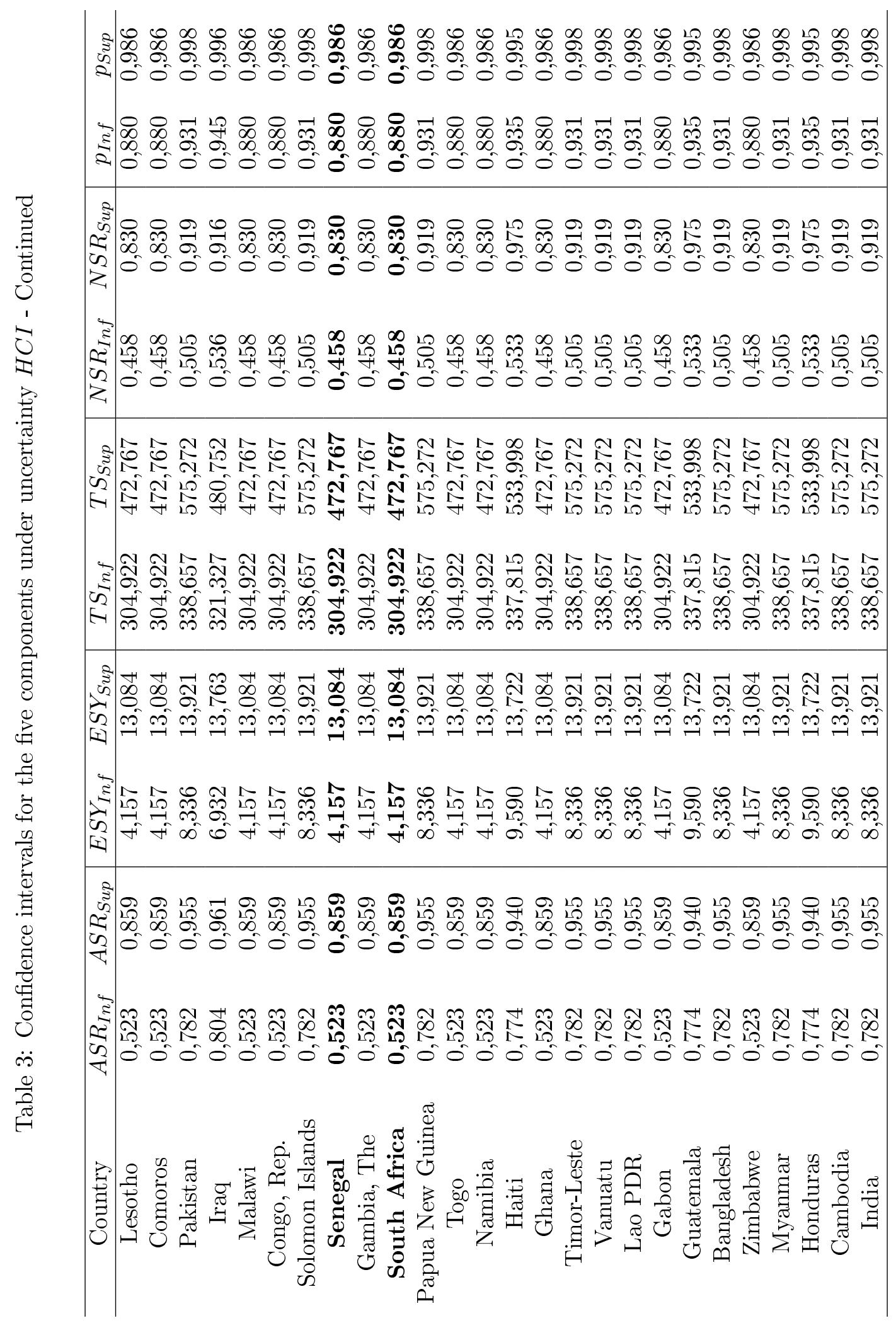




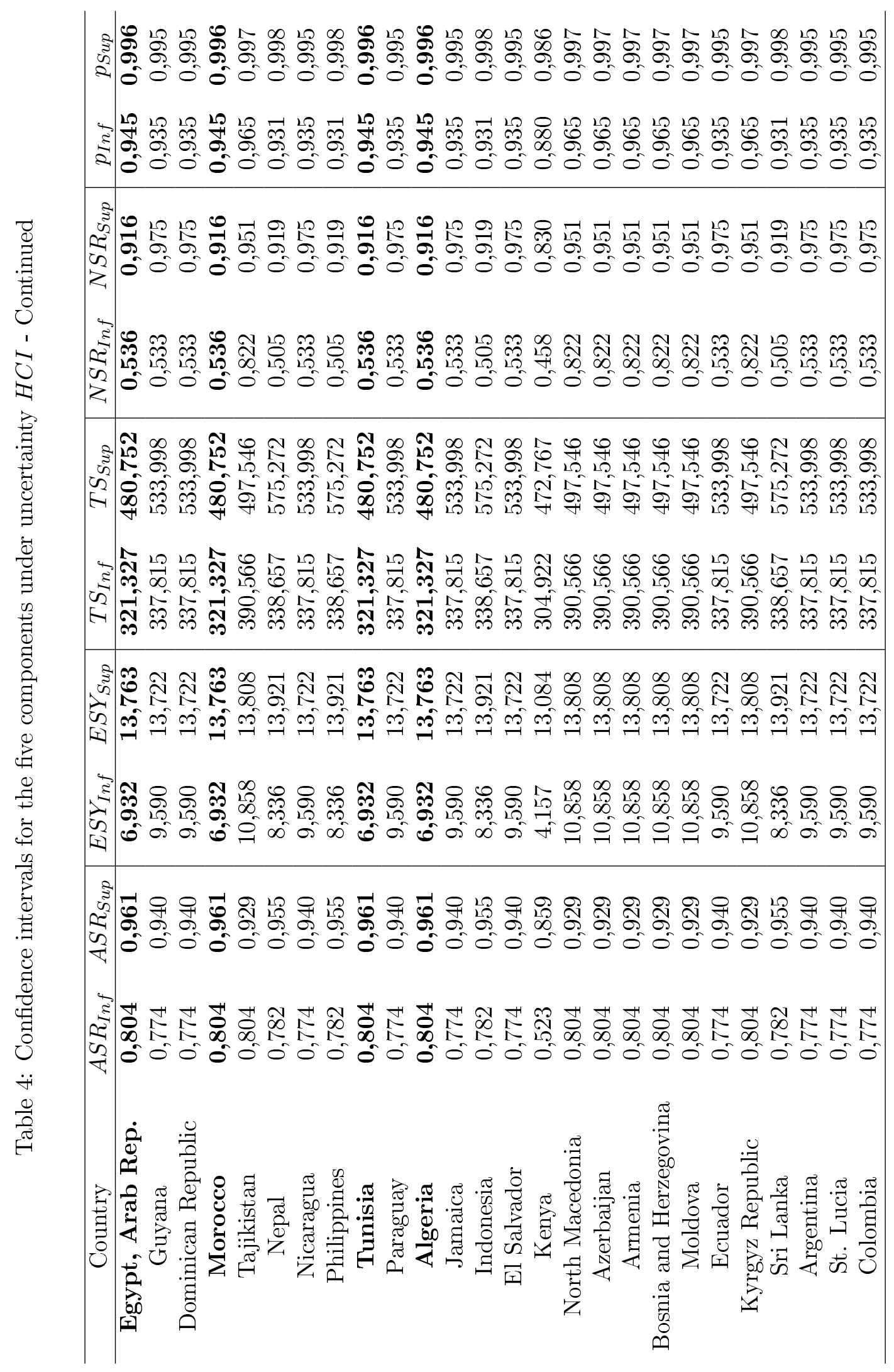




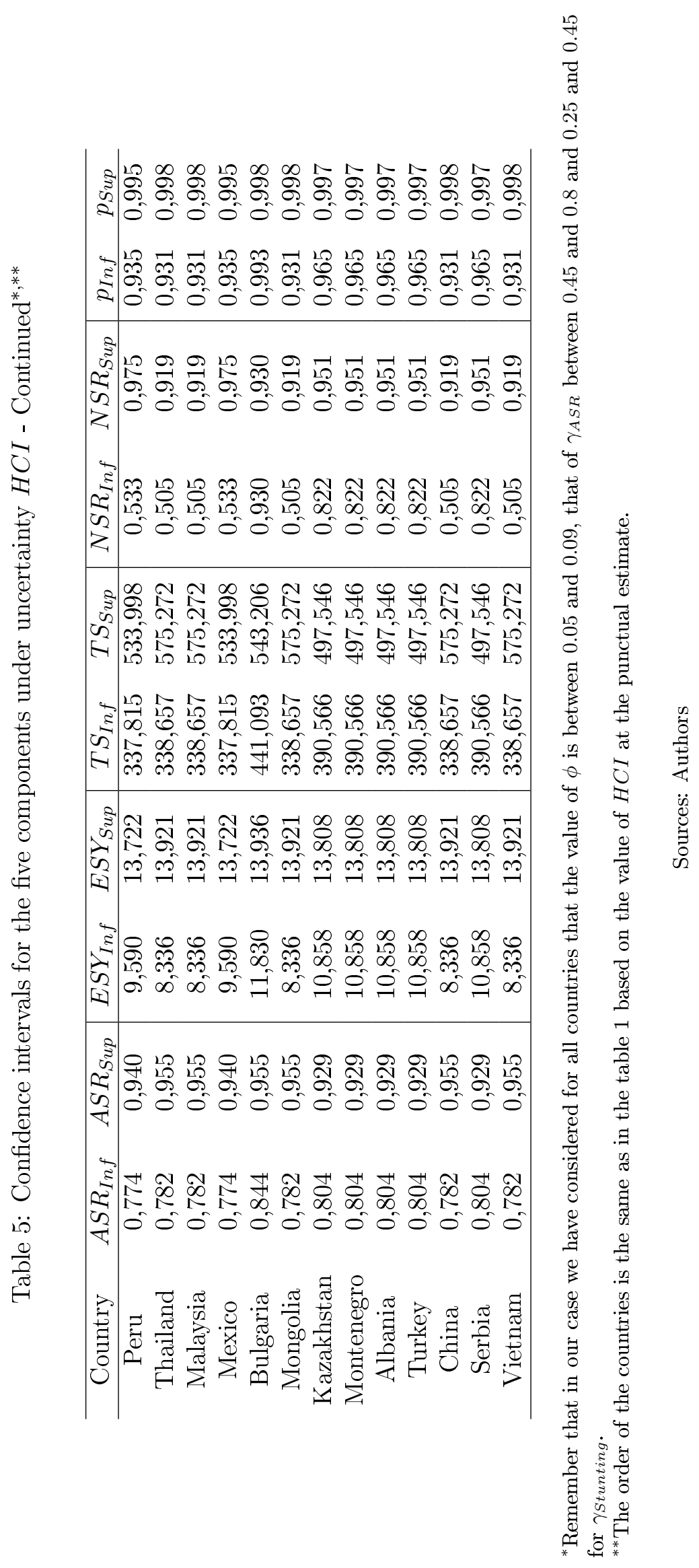

